\title{
La simpatía hacia los personajes de ficción: un examen de los factores implicados desde la psicología social y la teoría fílmica cognitiva
}

\section{Sympathy for fictional characters: an examination of the factors involved from a social psychology and cognitive film theory perspective}

Víctor Aertsen. Universidad Carlos III de Madrid (vaertsen@db.uc3m.es)

Recibido: 03/04/2017 - Aceptado: 10/05/2017

\section{Resumen:}

La simpatía es el principal vínculo afectivo que el espectador establece con los personajes de ficción, siendo un prerrequisito necesario para la elicitación de otras emociones. Dada su importancia, el objetivo del presente artículo es detallar los diferentes factores que participan en su constitución, en un intento de ahondar en su comprensión. Para ello se recurre a diferentes trabajos enmarcados en el ámbito de la psicología social y la teoría fílmica cognitiva, se detallan algunos recursos textuales con los que las narraciones audiovisuales explotan cada factor concreto, y se reflexiona sobre los efectos que cada uno de ellos tiene sobre la simpatía y el resto de factores que la fundamentan.

Palabras clave:

Identificación; simpatía; teoría fílmica cognitiva; personajes ficcionales; emociones narrativas.

\section{Abstract:}

Sympathy is the main affective bond that the viewer establishes with the fictional characters, being a necessary prerequisite for the elicitation of other emotions. Given its importance, the objective of this article is to detail the different factors involved in its constitution, in an attempt to delve into its understanding. To do it, we turn to different works framed in the field of social psychology and cognitive film theory, we describe some textual resources that audiovisual narratives use to exploit each particular factor, and we reflect on the effects that each of them has on sympathy and the other factors involved.

Keywords:

Identification; sympathy; cognitive film theory; fictional characters; narrative emotions. 


\section{Introducción}

Investigar el modo en que se establece y desarrolla la relación afectiva del espectador con los personajesde ficción es, según James Wood (2008: 171), participar en una de las dos discusiones más extensas sobre la narración dramática desde Platón y Aristóteles (siendo la otra la naturaleza de la mímesis ${ }^{1}$. Si bien en el ámbito de la teoría cinematográfica la primera corriente epistemológica que abordó el tema con sistematicidad fue la teoría psicoanalítica, en las últimas décadas la teoría cognitiva ha cogido el relevo, precisando y profundizando en la cuestión a partir del amplio corpus de investigaciones y reflexiones aportadas por la psicología social y la neurociencia sobre la materia.

A pesar de que autores como Alex Neill (1996), Berys Gaut (1999) o Amy Coplan (2009) defienden la innegable importancia de la 'empatía', la mayoría de autores adscritos a la teoría cognitiva consideran que el espectador se involucra emocionalmente en el devenir de los acontecimientos narrados en función, primordialmente, de su 'simpatía' con los personajes. Carroll (2008: 78) señala que la simpatía "constituye el mayor cimiento emotivo entre la audiencia y los personajes fílmicos relevantes". Y Plantinga (2009: 149) apunta que "la simpatía por un personaje lleva al espectador a mostrar especial interés en el bienestar del personaje y a desarrollar fuertes preocupaciones sobre los resultados de la historia". Si bien a veces complementada por procesos de imitación corporal y simulación imaginativa² ${ }^{2}$ la simpatía es el principal mecanismo por el que los espectadores se involucran emocionalmente en las vivencias de los personajes, preocupándose por el devenir de los acontecimientos narrados.

La simpatía es un estado emocional proyectado hacia el exterior, dirigido hacia otras personas, y que se define como la presencia de una inclinación afectiva hacia ellas. Según Carroll (2008: 179), "es nuestra simpatía por el personaje lo que

1 Popularmente se utiliza el concepto ‘identificación’ para hacer referencia a la relación cognitiva y emocional establecida entre personaje y espectador. Se trata de un término de uso generalizado tanto en el lenguaje coloquial como en la tradición filosófica occidental, asentado en los estudios fílmicos por el uso sistematizado que la teoría fílmica psicoanalítica haría de él. Dada la vaguedad con que el término es generalmente utilizado, su asociación con una corriente epistemológica cuyos postulados se rechazan y la problemática que erige su propia etimología (al sugerir una equivalencia o identidad entre espectador y personaje), los teóricos fílmicos cognitivos prefieren utilizar otros términos para referirse a la relación emocional entre espectador y personajes, como la expresión 'involucración con los personajes' (character engagement) (Smith, 1995).

2 Por lo general, la bibliografía especializada recurre al concepto de empatía para hacer referencia a dos tipos de procesos por los que un individuo (espectador) puede identificarse corporal, emocional y/o cognitivamente con otra persona (personaje), teniendo en cuenta que en este caso el término identificación resulta pertinente, dado que los procesos mencionados implican tomar la perspectiva experiencial del otro individuo. Primero, los procesos de 'empatía corporalizada' (Keysers, 2011), por los que el espectador se acopla o contagia del estado afectivo del personaje mediante mecanismos mentales automáticos tales como la imitación motora y somática, sin depender de formas superiores de cognición e imaginación. Segundo, la denominada 'empatía imaginativa' (Goldman, 2006), por la que el espectador se involucra emocionalmente con el personaje al imaginar o simular lo que debe ser estar en su situación, usando su imaginación para evaluar la situación del personaje desde el interior (Gaut, 2010) o centro (Smith, 1995) de ésta, lo que le lleva a sentir lo mismo que el personaje. Para una explicación más detallada de ambos procesos en el caso del cine, ver Vaage (2010). 
nos dispone a considerarlo parte de nuestra red de preocupaciones, y, por lo tanto, a evaluar una injusticia cometida contra él como algo perpetrado contra 'uno mismo'”. Implica una actitud positiva en su favor, que provoca reacciones viscerales de aflicción cuando los objetos de nuestra simpatía se enfrentan a problemas, y sentimientos de júbilo cuando consiguen mejorar su situación.

Si bien se trata de una emoción en ocasiones sentida en sí misma, entreverada con las emociones que la constituyen, generalmente funciona como una disposición emocional que suscita la emergencia de otras emociones que dependen de ella, en función del grado de preocupación que instaura respecto al bienestar de la persona considerada simpática. Son estas emociones dependientes de la simpatía (Plantinga, 2009) las que más interesan desde un punto de vista narrativo: el suspense, el júbilo, el miedo, la tristeza, la ira, etc. Pero esta dependencia explica la identificación de la simpatía como la disposición fundacional de la relación entre el espectador y el personaje. La simpatía "provee la óptica emotiva a través de la cual medimos la narración de una punta a la otra" (Carroll, 2008: 179), pues da forma a nuestra recepción emocional.

\section{Objetivos y método}

Dada su importancia, conviene examinar cómo se constituye la simpatía del espectador hacia los personajes. Por ello, en el siguiente apartado se proponen y describen una serie de factores que participan en la vinculación afectiva del espectador con los personajes de una ficción audiovisual. Para elaborar la lista de factores propuestos se ha tenido en cuenta aquellos factores planteados previamente por otros trabajos del ámbito de los estudios fílmicos, pero contrastastando y ampliando en todo caso las descripciones formuladas recurriendo a diferentes trabajos propios de psicología social, la rama de las ciencias cognitivas que ha privilegiado hasta el momento el estudio de las vinculaciones afectivas entre individuos.

Determinar la relevancia singular de cada factor a partir del análisis de diferentes obras cinematográficas resulta prácticamente imposible: todos ellos están interrelacionados, un mismo elemento textual suele participar en la activación de varios, y las narraciones siempre persiguen su acción combinada. Pero diferentes trabajos en el campo de la psicología social han analizado el efecto de cada una de ellas por separado, mediante experimentos destinados a su medición singular. Y una reflexión comparada en el ámbito de la teoría cinematográfica permite esclarecer su importancia relativa en las narraciones fílmicas. Por ello, a pesar de resultar imposibles de aislar en la práctica, su distinción teórica permite afinar el análisis de cada texto concreto, algo especialmente útil de cara al examen de obras donde la vinculación resulta controvertida (el espectador simpatiza con un personaje de cualidades morales cuestionables) o fallida (el espectador no consigue simpatizar con el personaje propuesto por el texto). 


\section{Resultados}

Partiendo del examen de diferentes trabajos científicos dedicados al análisis de los factores que participan en la vinculación afectiva interpersonal, y teniendo en cuenta la naturaleza mediada de la relación entre el espectador audiovisual y los personajes de ficción, este trabajo propone la definición de siete sentimientos que se consideran fundamentales en la constitución de la simpatía del espectador hacia las figuras que protagonizan los relatos audiovisuales: aprobación, admiración, compasión, atracción, familiaridad, homofilia e intimidad. Los siguientes apartados se dedican a la descripción pormenorizada cada, con el fin de comprender mejor el funcionamiento particular de cada uno y la interacción entre ellos durante el proceso de visionado.

\subsection{Aprobación}

Los teóricos cognitivos que han abordado el problema de la simpatía señalan la aprobación moral de los personajes -de sus actos, sus intenciones e incluso su 'fondo moral'- como el factor dominante en su constitución (Smith, 1995; Carroll, 1996, 2008; Plantinga, 2009). Primacía de lo moral que también defienden los autores enmarcados en la teoría de la disposición (Raney, 2004, 2011)3 y que se puede explicar por la propia naturaleza del enunciado narrativo, el cual, al articularse sobre unos conflictos interpersonales, inevitablemente suscita la tasación moral de acciones e intenciones. En cualquier caso, la aprobación moral de los personajes no es categórica, sino relativa. Todos los autores mencionados están de acuerdo en que la aprobación moral de cada personaje se establece en función de la posición que ocupa dentro de la estructura moral interna de la obra, donde estos valores son organizados en posiciones jerárquicas de relativa preferibilidad. Una estructura moral que varía a lo largo de la obra, y permite explicar el fenómeno de la "simpatía por el diablo" (Carroll, 2009; Smith, 1999, 2011), es decir, los casos en los que el espectador simpatiza con personajes de moralidad reprochable, como Tony Soprano en The Sopranos (Los Soprano, David Chase, 1999), al resultar generalmente más aprobable que aquellos personajes contra los que se enfrenta en cada temporada.

Por otro lado, el espectador flexibiliza sus principios morales durante el visionado audiovisual, pudiendo aceptar la estructura moral de la película en pos del placer y el entretenimiento, incluso cuando ese sistema entra en conflicto con sus creencias. En este sentido, Smith (1995: 189) advierte de una posible "suspensión de valores" cuando nos involucramos con películas de gángsters. Arthur Raney (2011) observa que el afán lúdico con que el espectador accede a las ficciones audiovisuales le motiva a obviar las exigencias cognitivas implicadas en la evaluación moral deliberada, produciéndose una "desvinculación moral en aras del disfrute". Y Margrethe Bruun Vaage (2013) habla sobre el "desahogo ficcional" de los

3 La teoría de la disposición se interesa por explicar cómo los individuos evalúan y forman afiliaciones con los personajes mediáticos, y cómo el disfrute se ve afectado por lo que sucede con y a esos personajes (Zillmann and Cantor, 1976), si bien abordan la cuestión desde el campo de la psicología, mediante estudios experimentales. 
espectadores, que se ven liberados de las obligaciones morales de su vida real (pensar en las consecuencias de los actos, prestar ayuda a los que la necesitan, etc.). Se entiende así que un espectador pueda encontrar placer en la violencia con la que Harry Calahan liquida a Scorpio en Dirty Harry (Harry el sucio, Don Siegel, 1971), cuando ese mismo comportamiento sería recriminado por el mismo espectador en la vida real. Violencia que contrasta y se ve relativizada al ser comparada con la del villano, de motivaciones menos nobles y actuar más sádico.

Durante el visionado, la aprobación moral no surge de un juicio objetivo y racional de la orientación moral del personaje, sino generalmente de una evaluación automática e inconsciente de esta. Esta se puede considerar, siguiendo los postulados de la corriente intuicionista de la filosofía y psicología moral ${ }^{4}$, como el resultado de una intuición, antes que una deliberación consciente y elaborada (Haidt, 2001; Haidt y Kesebir, 2010). Es decir, la aprobación no se fundamenta sobre criterios éticos, sino sobre una serie de suposiciones, prejuicios, sesgos e interferencias afectivas que hacen brotar una estimación rápida y superficial de la orientación moral del personaje, susceptible de múltiples matizaciones y modificaciones. En este sentido, la apreciación moral está dominada por el efecto de emociones básicas (como el asco, la ira), heurísticos morales (como el tabú social al incesto) y sesgos mentales (favoritismo, parcialidad) que, automatizados, funcionan como marcadores somáticos de lo moralmente aceptable o rechazable, distorsionando el juicio moral racional.

De estos sesgos, probablemente el más relevante sea el favoritismo. La aprobación moral está condicionada por el grado de simpatía que el personaje despierta en el espectador en el momento de ser evaluado, ya que "nuestra tendencia a excusar o defender acciones y motivaciones inconsistentes con nuestras expectativas incrementa con la fuerza de la intensidad disposicional hacia el personaje" (Raney, 2011: 148). Existe una notoria circularidad en el modo en que aprobación y simpatía se retroalimentan: la psicología social demuestra que tendemos a mostrar favoritismo en nuestras evaluaciones morales hacia aquellos individuos que nos son simpáticos (Haidt, 2012), probablemente a causa de la acción combinada del sesgo endogrupal y del sesgo de confirmación. Es más, generalmente mostrar favoritismo (lealtad, comprensión) hacia las personas que nos importan se considera moralmente loable, convirtiéndose culturalmente el desajuste en virtud.

La incidencia del favoritismo en la evaluación moral y la vinculación simpática con los personajes tiene diferentes consecuencias. Por un lado, avala la importancia de las predisposiciones con las que el espectador llega al visionado: una leve simpatía o antipatía previa hacia un personaje y/o quien lo interpreta puede condicionar futuras evaluaciones de su orientación moral (Raney, 2004). De forma similar, explica el efecto de las primeras impresiones sobre posteriores evaluaciones, y por tanto la importancia del orden ('efecto marco') en que los rasgos del personaje son presentados al espectador a lo largo de la narración (Appiah, 2008: 82-83).

4 La corriente intuicionista parte de una concepción dual de la cognición humana, en boga en las ciencias cognitivas contemporáneas, donde se diferencian dos rutas de procesamiento mental (Kahneman, 2012): una de carácter intuitivo (sistema 1), automática, veloz y sujeta a múltiples sesgos; y otra orientada a la deliberación (sistema 2), consciente, lenta y neutral. 
Finalmente, revela "lo testarudos que somos como simpatizadores", como señala Vaage (2014: 279), que recurre al favoritismo para explicar la simpatía del espectador con el protagonista de la serie Breaking Bad (Vince Gilligan, 2008) durante su cuarta temporada, en la que comete una larga serie de atrocidades imposibles de exonerar racionalmente. Por ello, según Raney (2011: 147), en muchos casos es más apropiado decir que "los espectadores leen o interpretan las acciones de los personajes simpáticos como moralmente apropiados en aras de la conservación y defensa de sus actitudes positivas hacia estos, en lugar de evaluarlas moralmente en función de su adecuación". A medida que avanza la narración, más que determinar la simpatía hacia un personaje a partir de su aprobación, el espectador aprueba al personaje en función de su simpatía hacia él.

En cualquier caso, la aprobación moral de los personajes por parte del espectador está condicionada por su propio sistema moral, que no solo rige sus evaluaciones morales deliberadas, sino especialmente las de carácter intuitivo. Toda narración se construye teniendo en mente un 'sistema de valores' ideal o 'co-texto', que Smith (1995: 195) define como el “conjunto de valores, creencias y demás que forman el telón de fondo de los eventos de la narración”, es decir, "el contexto dentro del texto" para el que fue creada y en el que se espera sea recibida (y evaluada) la película, para funcionar según las intenciones de sus autores. En obras cercanas en el tiempo y el espacio, el co-texto es habitualmente invisible, en tanto que se adecúa a los valores del mundo social en el que vive el espectador. Pero el tiempo o su recepción transcultural hacer evidente un 'co-texto' que no coincide con el sistema de valores del espectador.

Smith (1995) plantea The Birth of a Nation (El nacimiento de una nación, D. W. Griffith, 1915) como ejemplo paradigmático de un co-texto de valores que chocan con los nuestros, donde la amabilidad de Austin Stoneman hacia los personajes afroamericanos es asumida por la película como cargada negativamente, del mismo modo que el comportamiento paternalista de la familia Cameron hacia sus sirvientes afroamericanos es aprobado (en el mejor de los casos, según el co-texto de valores de la película, Stoneman es moralmente débil; en el peor de los casos, moralmente corrupto, el 'malvado' dentro del sistema moral del texto).

Si bien la aprobación moral no es el único factor implicado en la simpatía, constituye, como apunta Smith (2011: 84), "una especie de centro de gravedad que los factores amorales pueden modular, pero no desplazar". La aprobación moral no solo es el factor más importante per se, sino que además se ve afectado directa o indirectamente por todos los demás factores. Resulta por ello complicado encontrar casos en los que la película elicita una intensa simpatía hacia sus personajes en base a factores amorales. Precisamente con el propósito de circunvalar la importancia de la aprobación moral los estudios sobre la vinculación afectiva con los personajes recurren habitualmente a textos donde el espectador se vincula con un personaje en principio moralmente cuestionable, y que por lo tanto debe presentar otras características que eliciten su simpatía. 


\subsection{Admiración}

Los autores antes citados extienden la evaluación moral de los personajes a rasgos de su personalidad, siguiendo una perspectiva propia de la ética de la virtud ${ }^{5}$. Se entiende así, por ejemplo, que Carroll (1996) destaque la valentía y el pragmatismo de los protagonistas de Le salaire de la peur (El salario del miedo, Henri Georges Clouzot, 1953) a la hora de evaluarlos moralmente. Virtudes susceptibles de despertar admiración, en tanto que personaje y espectador comparten un mismo espacio cultural donde estos rasgos son apreciados. Por tanto, los personajes consiguen la simpatía del espectador demostrando su rectitud moral, su deseo de hacer el bien. Pero también exhibiendo rasgos de personalidad loables, como fortaleza, ingenio, valor, templanza o perseverancia; y habilidades y conocimientos dignos de admiración, sean destrezas atléticas, pericia técnica o erudición cultural.

La psicología social predice que la admiración es un factor importante en la simpatía (Huston, 2013). Pero a su vez advierte contra la perfección. No solo por la importancia de su vulnerabilidad en la mecánica del suspense narrativo (Carroll, 1996). Sino porque, como demuestra el "efecto Pratfall", la simpatía hacia un personaje admirable aumenta cuando este comete algún error ocasional o presenta algún defecto menor en su personalidad, al dotarle de una mayor humanidad (Aronson, Willerman y Floyd, 1966). Ya lo advertía Aristóteles (2006: 1453a), al recomendar que la tragedia debía estar protagonizada por "un hombre no virtuoso en extremo".

Se podría argumentar que una ligera mella en la personalidad y capacidad del personaje tiene efectos positivos sobre la simpatía. No solo por el "efecto Pratfall", sino porque la propia admiración puede dejar de tener un efecto significativo llegados a determinado punto, resultando más lucrativo para la narración que el personaje produzca otro tipo de sentimiento en el espectador. Una aplicación extendida aunque intuitiva de esta teoría en el cine se puede observar en el modo en que los héroes del cine de acción son habitualmente caracterizados con infinidad de rasgos admirables pero una vida romántica desastrosa, como ocurre con los problemas matrimoniales del protagonista de Die Hard (La jungla de cristal, John McTiernan, 1988). Y a la inversa, siguiendo el mismo razonamiento, no es de extrañar que el protagonista de Van Damme's Inferno (John G. Avildsen, 1999) consiga despertar menores simpatías en el espectador que los antes citados, teniendo en cuenta el modo en que la narración intenta presentarle como un personaje poderoso en todas sus dimensiones vitales, incluido en el ámbito sexual.

Obviamente, las virtudes ayudan a exonerar moralmente los actos reprochables de los protagonistas, por vía del favoritismo. Curiosamente, uno de los recursos más habituales en el cine criminal es proponer la adhesión de sus delictivos protagonistas a algún código moral cuyo férreo seguimiento es percibido por el espectador como una virtud laudable

5 Donde la moralidad no depende de la evaluación de las intenciones y acciones de los personajes en función de unas reglas absolutas (deontología) o consecuencias relativas (consecuencialismo), sino de la valoración de su personalidad a partir de su adecuación a unos rasgos socialmente alabados (virtudes). 
que, además, suele distinguirle de sus contrincantes, como el ladrón que no roba a los pobres (Parker, Taylor Hackford, 2013). Normas establecidas en el ámbito de la actividad criminal y que, paradójicamente, su exhibición parece exonerar parcialmente la criminalidad de sus personajes.

\subsection{Compasión}

La compasión es uno de los sentimientos centrales sobre los que se cimenta la simpatía en toda narración ${ }^{6}$. Ya en su Poética, Aristóteles (2006: 1453b) presentaba la piedad y el temor como las dos emociones básicas de la tragedia, instaurando la compasión una preocupación sobre la que se fundamentará el consabido temor (Smith, 2011).

Resulta habitual que los protagonistas de una película se presenten como víctimas de una situación o unas acciones lacerantes susceptibles de despertar la compasión del espectador. El abanico de percances es amplio, e incluso podemos sentir compasión por un personaje imposible de admirar, como ocurre con el protagonista de A Serious Man (Un tipo serio, Ethan Coen y Joel Coen, 2009), un profesor desaliñado de mediana edad que hace frente a sus problemas con una pasividad y confusión desalentadoras, pero que aun así, a causa de los desplantes y abusos a los que es incesantemente sometido, se granjea la compasión del espectador. En cualquier caso, la importancia de este sentimiento seminal va más allá de la anécdota biográfica, extendiéndose por toda la narración: al construirse todo relato sobre el conflicto, la serie de obstáculos a los que se enfrentan los protagonistas revelan sus vulnerabilidades y frustraciones, despertando algún grado de compasión en el espectador.

Varios factores influyen en la suscitación e intensidad de la compasión. Por un lado, la propia simpatía influye significativamente sobre la compasión, estableciéndose una correlación positiva entre ambos sentimientos (Gabriel, 2015): los personajes más simpáticos son susceptibles de producir más compasión cuando se muestran en problemas. Por otro lado, la vulnerabilidad intrínseca (discapacidad, fragilidad) percibida en la víctima influye de forma destacada sobre la compasión. En este sentido, Loewenstein y Small (2007) sugieren la existencia de un posible heurístico moral que condiciona la compasión que produce una víctima en función de su edad y sexo, resultando mayor cuando se trata de una mujer o un niño. Se trataría de un sesgo desarrollado por la enculturación de los individuos en una sociedad donde se

6 En ocasiones resulta complicado distinguir en la bibliografía especializada cuándo se habla de simpatía y cuándo de compasión. Una confusión fomentada en inglés por la polisemia del término sympathy, cuya primera acepción es equivalente a lo que en castellano se entiende por compasión (sentimiento de conmiseración hacia quienes sufren), y su segunda acepción hace referencia a lo que en castellano se identifica con la simpatía (sentimiento de cariño y afinidad), sensación a la que los angloparlantes suelen referirse con el término liking. Así, por ejemplo, Plantinga (2010: 41) utiliza el término para hacer referencia al sentimiento "es provocado por el trato injusto percibido, la necesidad de protección y factores no-morales similares”. En cambio, tanto Carroll (1996, 2008, 2009) como Smith $(1995,1999,2011)$ suelen considerar la simpatía en un sentido más amplio, como demuestra no solo el conjunto de sentimientos que según ellos la constituyen (piedad, fascinación, aprobación moral, etc.), sino la serie de términos que en cierto sentido usan como sinónimos de esta: affinity, loyalty, appealing o liking. 
hace reiterada alusión a su vulnerabilidad, y que el cine explota habitualmente. Especialmente el cine de terror e intriga, dado a poner en peligro a personajes femeninos.

A ello se añade que la compasión hacia un personaje depende de la capacidad del espectador de empatizar con él. Es decir, el espectador primero debe ponerse en el lugar del personaje para comprender y compartir el sufrimiento que la situación le produce, para luego poder compadecerle desde una posición externa. Loewenstein y Small (2007) resumen una serie de factores que influyen en la elicitación de la compasión, cuatro de los cuales dependen directamente de la empatía.

En primer lugar, la importancia de las experiencias previas del espectador. Que el observador haya experimentado directa o vicariamente -a través de algún conocido cercano- situaciones similares a las del personaje aumenta la intensidad de la empatía suscitada por estas y, en consecuencia, la compasión. Lo que incluye compadecer intensamente a un personaje que sufre alguna agresión o pérdida traumática (Barnett, Tetreault y Masbad, 1987; Christy y Voigt, 1994). Pero también sentir cierta lástima por sus dificultades cotidianas, a las que pueden remitirse todos los espectadores. Smith (2011: 84) defiende que la simpatía del espectador hacia Tony Soprano radica precisamente en su caracterización como "un tipo cualquiera" cuyas dificultades diarias nos llevan a "compadecer sus ansiedades, su vulnerabilidad y sus frustraciones cotidianas".

También influye la intensidad sensorial de la situación contemplada. Cuanto más vívida sea la situación desde una perspectiva audiovisual, más efectiva la empatía y mayor la compasión. Las investigaciones en el ámbito de la neurociencia (neuronas espejo) demuestran que la observación directa de un rostro que expresa sufrimiento o un cuerpo que sufre una agresión produce una reacción corporal inmediata en el espectador, de similiar cualidad pero reducida intensidad (Keysers, 2011). Lo que explica la habitual presentación del rostro de los protagonistas con fines empáticos (Plantinga, 1999), un fenómeno extensible a sus cuerpos heridos. Pero la viveza de la experiencia no solo tiene que ver con su presentación audiovisual, sino también con su imaginación. La psicología social demuestra que la empatía hacia el personaje puede magnificarse cuando el observador ha sido primado para tomar la perspectiva de la víctima, es decir, cuando no solo observa la situación sufrida por la víctima, sino que además se le ha invitado a imaginarse activamente en esa misma situación (Bateson, et al., 2003).

En sentido inverso, la narración pueda fomentar la aprobación moral de alguno de sus personajes regulando la presentación de sus actos más reprochables, así como de sus consecuencias. Como plantea el dicho popular: 'ojos que no ven, corazón que no siente'. La omisión del sufrimiento ajeno provocado por sus estafas ayuda, por ejemplo, a que el espectador simpatice con el protagonista de The Wolf of Wall Street (El lobo de Wall Street, Martin Scorsese, 2013). Hecho que se puede explicar, además, a partir del 'efecto de la víctima identificable', según el cual los individuos tienden a sentir una mayor compasión hacia personas concretas e identificables que hacia grupos vagamente definidos (Small y Loewenstein, 2003). 
En tercer lugar, resulta importante el grado de coincidencia somática y patémica entre la situación del espectador justo antes de contemplar la escena en cuestión, y la del personaje durante involucrado en ella (Boven et al., 2013). Que el espectador llegue a la escena en un estado de tristeza, hambre o fatiga, por ejemplo, intensifica la compasión elicitada por una situación donde el personaje sienta esas mismas experiencias. La narración obviamente no puede prever el estado con que el espectador llega a la sala de cine, pero puede orientar sus experiencias somáticas y patémicas a lo largo de la obra, consiguiendo, por ejemplo, que simpatice intensamente con un personaje amado por el protagonista antes de que este sea asesinado, complementándose el dolor del personaje con el del propio espectador. En definitiva, la película puede dirigir las reacciones del espectador en pos de un primado experiencial que asemeje su estado al del personaje, intensificando la compasión.

Finalmente, la novedad de la situación concreta para el espectador es otro factor importante. Si bien cada nueva exposición al sufrimiento ajeno suscita compasión, a medida que el número de exposiciones aumenta la intensidad de cada reacción se ve gradualmente atenuada. Este fenómeno, conocido como 'exposición gradual' (Glover, 2001), resulta central para explicar la progresiva insensibilización de los individuos ante el dolor ajeno, tanto en determinados contextos históricos (las atrocidades del nazismo) como en entornos controlados (el famoso experimento de Milgram). Desde el punto de vista de la narración cinematográfica, la exposición gradual predice la progresiva ineficacia de presentar situaciones en las que el personaje se ve sometido una y otra vez a percances de similar naturaleza.

La compasión tiene fuertes efectos sobre la aprobación moral de los personajes. Que los protagonistas muestren compasión hacia otros personajes más vulnerables es una de las estrategias más recurrentes para granjearse la aprobación moral de los espectadores (Carroll, 1996). Y, a la inversa, cuando los antagonistas victimizan a otros personajes elevan a los protagonistas en la estructura moral del texto (Smith, 1999). Por otro lado, que los protagonistas se enfrenten a situaciones lacerantes no solo despierta nuestra piedad, sino que les permite demostrar cuán capaces son de gestionar el dolor y las emociones adversas, y, por lo tanto, exhibir virtudes como la templanza, la disciplina o la prudencia. De ahí el habitual éxito del estoicismo ante el dolor de los héroes cinematográficos.

Por último, la extereorización de sentimientos morales como la vergüenza y sobre todo la culpa también actúa positivamente sobre la aprobación moral de los personajes, un recurso especialmente lucrativo en el cine criminal, donde es habitual que los remordimientos del protagonista ayuden en la exoneración de sus execrables actos a ojos de los espectadores. Es el caso de Tony Soprano, cuya culpabilidad, según Smith (2011: 77), demuestra que es un ser moral, en tanto que "moralmente sensible". 


\subsection{Atracción}

Junto a la apreciación moral, un segundo factor relevante en la simpatía es la atracción prosocial que el personaje produce, es decir, su capacidad de seducir, agradar o divertir al espectador. En primer lugar, la atracción sexual implica una evaluación positiva de carácter erótico, fundamentada en el deseo sexual hacia el otro. Entre los rasgos que se toman en consideración cabe mencionar, esencialmente, su aspecto físico, su voz, el modo en que se mueve y su personalidad. Se trata de un sentimiento entretejido con la aprobación o la admiración, pero de dinámica diferente.

En este sentido, el cine comercial ha recurrido tradicionalmente al poder seductor del físico y la personalidad de sus estrellas para establecer vínculos afectivos entre el espectador y los personajes que estos encarnan, diseñados habitualmente con la intención de potenciar la atracción. La belleza, la sensualidad, el carisma o el garbo de los personajes, además, condicionan positivamente la apreciación moral del personaje, como demuestran los estudios empíricos en torno al llamado 'efecto halo', un sesgo cognitivo que lleva a extender la percepción positiva -o negativa- de un rasgo sobre otros con los que no tiene una relación directa.

Múltiples investigaciones han confirmado que el atractivo físico es la variable que más evoca el efecto halo, lo que conlleva que alguien percibido como atractivo debido a sus rasgos físicos sea percibido como una persona más inteligente, generosa, honesta o exitosa, independientemente del sexo tanto del sujeto evaluado como del observador. Además, más allá de su personalidad, se ha demostrado que el efecto halo tiene consecuencias en la evaluación moral de las acciones de los individuos, encontrándose en un estudio que las personas percibidas como más atractivas suelen ser sentenciadas a condenas menores que las personas poco atractivas, aun habiendo cometido el mismo crimen (Efran, 1974). Si bien un factor menor, el atractivo físico de los personajes inclina ligeramente al espectador hacia su aprobación moral.

Junto a esta dimensión erotizada de la atracción cabe situar otra de carácter afable: la ternura. Se trata de un sentimiento prosocial que lleva al espectador a considerar el objeto de su ternura como un ser dulce, delicado, digno de afecto, cariño y cuidados. Numerosos estudios en psicología social apuntan que los espectadores están predispuestos a simpatizar con personajes cuyos rasgos faciales se adecúan a lo que el etólogo Konrad Lorenz describió como 'esquema infantil' (cabeza grande, frente ancha, ojos redondos y amplios, nariz pequeña, mentón pequeño, mejillas redondas, etc.). El 'esquema infantil' no solo motiva sentimientos de cariño y preocupación hacia el sujeto, sino que además influye positivamente en su percepción social, siendo valorado como más agradable, amistoso, sano y competente (Glocker et al., 2009).

En este sentido, tiene efectos sobre otros factores como la aprobación moral, que favorece, o la compasión, que intensifica. Se trata de un factor explotado habitualmente en el cine de animación, sobre el que Gould (2010) o Etcoff (1999) han reflexionado para los casos de Mickey Mouse y Bambi, respectivamente. Y que ha sido usado en el cine de ficción no solo para asegurar simpatías, sino también para complicar la antipatía de los espectadores hacia los antagonistas del 
drama, como brillantemente plantea ¿Quién puede matar a un niño? (Narciso Ibáñez Serrador, 1976). Al igual que a sus protagonistas, una pareja de turistas que visitan una isla mediterránea donde los niños han asesinado a todos los adultos del lugar, el esperable recelo que durante toda la trama deberían producir los infantes del pueblo en el espectador se ve obstaculizado por la inevitable ternura que sus rostros inducen, bloqueando sospechas y acciones en su contra. Beneplácito que posteriormente intensificará la sensación de alevosía y desprecio hacia los jovencitos, cuando se les observa cometer actos atroces explícitamente.

Finalmente, la gracia o vis cómica resulta otro factor importante. Primero porque la capacidad de divertir al espectador, de hacerle reír, resulta un mecanismo poderoso para granjearse sus simpatías. Y segundo, porque su comicidad aligera la apreciación moral de sus intenciones y acciones, exonerando al personaje de un juicio moral de otro modo más férreo (Jones, 2011). Personajes que no destacan por la moralidad de sus actos e intenciones, ni siquiera en comparación con el resto de personajes de la obra, pero aun así nos resultan divertidos o atractivos, en ocasiones reciben nuestra simpatía a lo largo de la narración, como ocurre con Royal Tenenbaum (Gene Hackman) en The Royal Tenenbaums (Los Tenenbaums: Una familia de genios, Wes Anderson, 2001), cuya desfachatez y vileza a lo largo de la obra, donde engaña a casi todos los personajes e incluso insulta a varios, se ve aliviada por la gracia que nos producen sus comentarios.

\subsection{Familiaridad}

La familiaridad con el personaje puede ser otro factor relevante en la simpatía, si bien cabe distinguir diferentes tipos de familiaridad. En primer lugar, la mera familiaridad perceptiva con el personaje puede actuar positivamente sobre la simpatía que este produce en el espectador, como predice el 'efecto de mera exposición' o 'principio de la familiaridad', estudiado por la psicología social (Zajonc, 2001). Demostrado con objetos tan diversos y en principio neutros como caracteres chinos o figuras geométricas, se trata de un fenómeno psicológico por el cual las personas tienden a desarrollar una preferencia por aquello con los que se familiarizan perceptivamente. De lo que se deduce que el espectador tiende a simpatizar ligeramente más con los personajes más expuestos en pantalla.

En segundo lugar, la familiaridad transtextual del espectador puede llevar a que establezca sus simpatías a partir de factores externos al propio texto. Se trata de una serie de predisposiciones hacia los personajes y los actores que los encarnan que no dependen del modo en que son caracterizados por el propio texto, sino de expectativas y vinculaciones previas. En el caso de las secuelas, remakes y adaptaciones el espectador habitualmente se lanza al visionado manteniendo cierta vinculación afectiva previa con el personaje, establecida en anteriores encuentros, y que le predispone a simpatizar o antipatizar con el personaje (familiaridad intertextual).

Por otro lado, el espectador también arrastra a la sala de cine sus simpatías y antipatías hacia los actores que encarnan a los personajes en la ficción, predisponiendo la valencia del vínculo que establecerá con ellos (familiaridad extratextual). 
Incluso aquellos actores de los que el espectador no tiene una opinión formada provocan efectos sobre la simpatía del espectador, aunque solo sea por medio de la familiaridad perceptiva que inducen. Un fenómeno que explica, en parte, la importancia de las estrellas en la industria cinematográfica, dado su impacto en la respuesta emocional del espectador?

A lo que cabe añadir una posible predisposición a simpatizar directamente con el personaje que se reconoce como protagonista de la historia por el mero hecho de ser protagonista (familiaridad architextual). Que el protagonista actuará de forma virtuosa y el antagonista de forma reprochable es una de las expectativas narrativas más comunes, resultando un prejuicio que puede condicionar la simpatía y antipatía del espectador antes incluso de que este pueda evaluar otros rasgos de los personajes más importantes (Raney, 2004). Cabe especular que estas predisposiciones transtextuales, si bien resultan influyentes al principio de la obra, menguan significativamente en importancia a medida que esta avanza. Pero aun así pueden condicionar las evaluaciones ulteriores, dada la tendencia humana a interpretar las acciones de las personas simpáticas con favoritismo.

En tercer lugar, cabe señalar la importancia de la familiaridad motivacional con el personaje, es decir, el grado de comprensión de sus motivaciones al que el texto nos ha permitido acceder. La comprensión tiene especiales efectos sobre la aprobación moral del personaje. El propio Smith (1995: 223) admite que "una imagen desarrollada de las motivaciones así como de las acciones de un personaje [...] puede servir para exonerar acciones que, vistas en aislamiento de esta imagen más grande, pueden ser fáciles de condenar". Un fenómeno que Wayne Booth (1961: 245-246) denomina 'simpatía del infiltrado'.

Al saber más de los personajes y el contexto en el que se inscriben sus acciones, estamos más preparados para comprenderlos y, en consecuencia, a ser comprensibles con ellos. La psicología moral ha realizado diferentes estudios al respecto, encontrando habitual el favoritismo y la parcialidad hacia aquellas personas cuya historia se conoce. Por ejemplo, Kwame Anthony Appiah (2008: 104) plantea el siguiente caso:

Sabemos, también, cuán fácilmente una historia atractiva puede vencer nuestra lealtad a esta o aquella máxima. ¿ Deben los coches aparcado ante una boca de incendio, tal como el que pertenece a Bob el contable, ser retirado? Por supuesto. Ahora cuéntame una historia acerca de Joanna, una buena mujer que está teniendo un mal día, con detalles sobre sus esperanzas y sueños, su bondad a un amigo enfermo, su preocupación por un hijo con problemas. No quiero que su coche sea retirado.

7 El éxito global del cine de Hollywood no solo radica en sus agresivas estrategias de distribución, sino también en su capacidad de haber convertido a sus actores más destacados en estrellas internacionalmente conocidas. Un ejemplo perfecto de lo que Joseph Nye (2004) denomina 'poder blando'. 
Conocer solo un lado de la historia sesga nuestra aprobación moral, un fenómeno que Kahneman (2012: 86-87) etiqueta como el principio ‘lo que vemos es lo que hay'. Estar familiarizado con la historia de la otra persona contrarresta el 'error fundamental de atribución' o 'efecto de sobre-atribución', es decir, la tendencia o disposición a sobredimensionar disposiciones o motivos personales internos cuando se trata de explicar un comportamiento observado en otras personas, dando poco peso por el contrario a motivos externos, tales como el rol social o la situación, para este mismo comportamiento. Por ejemplo, consideramos la acción de Bob el contable como el fruto de su arrogancia e indiferencia hacia las normas, mientras que en el caso de Joanna evaluamos la misma acción como el resultado de la difícil situación de una persona eminentemente bondadosa. Conocer las motivaciones del infractor moral, siempre y cuando estas no desvelen una intencionalidad puramente inmoral, nos llevan a resultar indulgentes. Y en muchos casos, además, inducen compasión.

El favoritismo producido por la familiaridad motivacional es un fenómeno habitual durante el visionado de productos audiovisuales. Por ejemplo, Vaage (2014: 270-271) reflexiona sobre una escena de Los Soprano en que Tony intercepta y amenaza en un campo de golf al oncólogo de su tío, que ni contesta ni devuelve las insistentes llamadas de ambos. Según la autora, desde una perspectiva moral el comportamiento de Tony es reprochable, pero la serie se ha ocupado de que conozcamos sus motivaciones (la preocupación por el bienestar de su tío, los frustrados intentos de ponerse en contacto con el médico), mientras que del doctor no sabemos nada (más allá de su silencio, y el hecho de encontrarlo jugando al golf).

\subsection{Homofilia}

La homofilia o atracción por los iguales también influye sobre la simpatía hacia los personajes, si bien cabe distinguir diferentes formas de entender esta inclinación positiva hacia lo similar. Por un lado, la homofilia social hace referencia a la inclinación hacia otras personas que ostentan rasgos sociales parecidos a los del sujeto, como pueden ser la edad, el sexo, la raza, la clase o la orientación sexual. Se trata de un fenómeno que, más por intuición que por constatación, la teoría psiconalítica y los estudios multiculturalistas defienden como central en los procesos de identificación con los personajes (y sus perversiones). Y que en parte apoyan algunas investigaciones desarrolladas en el marco de la teoría cognitiva (Raney, 2004; Eder, 2006), que se fundamentan sobre lo que en psicología social se denomina 'sesgo del endogrupo' y la 'atracción intragrupal' (Berreby, 2008).

La homofilia no solo establece un vínculo afectivo con el personaje, sino que además puede influir ligeramente en su aprobación moral, especialmente a la hora de justificar el comportamiento reprochable de este (Schiller, Baumgartner y Knoch, 2014). Pero a pesar de su posible impacto, la mera similitud con el personaje no es un buen predictor de la vinculación afectiva con el personaje (Cohen, 2006), resultando indudablemente un componente menor en la constitución de la simpatía. 
Mucho más importante resulta la homofilia disposicional, es decir, la similitud en cuanto a las experiencias, los valores y las actitudes. En una investigación ya clásica, Zillmann y Cantor (1976), utilizando chistes que implicaban relaciones entre superiores y subordinados (padres-hijos, profesores-estudiantes, empleadores-empleados), encontraron que los individuos cuyas experiencias vitales presentes eran más cercanas a las del grupo de los superiores disfrutaban más de las bromas donde la víctima era el subordinado, y viceversa, sin importar sus rasgos sociales. El efecto de la homofilia disposicional se explica, en primer lugar, porque la convergencia facilita que el espectador entienda mejor las motivaciones y reacciones de los personajes, lo que no solo asegura una mayor inmersión en el fluir de la narración, sino que además aumenta la familiaridad motivacional con este, así como las posibilidades de empatizar con él.

Pero además de estos efectos indirectos, la homofilia disposicional actúa directamente sobre la simpatía por dos vías. En primer lugar, al fijar una sensación de adhesión o compañerismo con el personaje (Eder, 2006: 71), dada la congruencia de preocupaciones e intereses en relación a los acontecimientos narrados. Al compartir objetivos dentro de la narración, el espectador se preocupa por el bienestar y el éxito del personaje, lo que fecunda la simpatía hacia este. En segundo lugar, actúa positivamente sobre la simpatía por efecto del llamado 'sesgo de confirmación', según el cual los individuos tienden a favorecer aquellas informaciones y personas que confirman las propias creencias. De modo que no solo se alían narrativamente con estos personajes, sino que el mero hecho de que presenten perspectivas vitales similares les acerca a ellos, al acercarles a sí mismos.

La distinción entre homofilia social y disposicional resulta esencial, ya que permite ahondar en el análisis de las complejas y poliédricas reacciones de los espectadores a las narrativas audiovisuales, más allá de reduccionismos basados en la raza o el sexo, en los que ocasionalmente han caído algunas propuestas de los estudios multiculturalistas y feministas. Por ejemplo, ayuda a explicar el éxito de las películas de kung-fu hongkonesas de los años 1970s, con la obra de Bruce Lee a la cabeza, en países como EE.UU. o Japón, donde estas narrativas serían disfrutadas -siguiendo una lectura preferentepor un publico joven que encontraba en la rebeldía contra la autoridad demostrada por los protagonistas un elemento experiencial en el que anclar sus simpatías (entre otros), más allá de su identidad racial (a pesar de que los villanos de los filmes habitualmente eran japoneses o norteamericanos).

Finalmente, cabe señalar el efecto de la sincronía de los estados corporales del espectador y el personaje sobre la simpatía, un fenómeno que se podría denominar empatía corporal u homofilia corporal. Como se ha comentado, el grado de coincidencia entre el estado somático y patémico del espectador con el personaje durante una escena influye sobre su empatía cognitiva y emocional con este, lo que tiene consecuencias para la compasión. Pero la empatía corporal con el personaje también tiene efectos directos sobre la simpatía, como demuestran diferentes investigaciones centradas en el fenómeno de la imitación motora y emocional (Chartrand y van Baaren, 2009). De modo que el seguimiento espacio-temporal continuado de un personaje, así como una intensa actividad corporal de estos, influyen sobre la simpatía. 


\subsection{Intimidad}

Por último, el grado de intimidad establecido con el personaje también puede ser un factor con cierto impacto sobre la simpatía hacia este. Por intimidad, nuevamente, se puede considerar múltiples aspectos.

Intimidad proxémica, en primer lugar, en tanto que cercanía puramente espacial. La proximidad visual al personaje tiene consecuencias sobre otros factores, como la familiaridad y la homofilia corporal. Pero también puede alentar inconscientemente una sensación positiva de intimidad al permitir el acceso a su espacio personal, una proximidad a la que en la vida real solo acceden las personas con las que se tiene una relación más cercana (Eder, 2006: 72-73). Pero intimidad proxémica también puede producir el efecto contrario, en caso de un uso excesivo, como ocurre en Keane (Lodge Kerrigan, 2004), donde los incesantes primeros planos sobre el protagonista terminan por producir una sensación de incomodidad, dada la invasión incesante del espacio corporal ajeno, no tanto por parte del espectador hacia el personaje como viceversa.

También cabe mencionar una dimensión de la intimidad analizada empíricamente, y que se podría denominar intimidad interaccional. En literatura, se ha demostrado que la actividad narrativa en primera persona por parte de un personaje incrementa la vinculación afectiva con este (Nodelman, 1991), conclusión fácilmente extensible al caso de la narración audiovisual. Por otro lado, como demuestran las investigaciones en el ámbito de la 'interacción parasocial' (PSI) ${ }^{8}$, cuando los personajes tratan a los espectadores como si estuvieran participando en una interacción social real, por ejemplo al saludarles o dirigirse a ellos directamente, el grado de vinculación afectiva aumenta (Auter, 1992). Se trata de un recurso habitual en la historia del teatro, que el realismo desestimó, pero que en la actualidad resulta cada vez más habitual en las narrativas audiovisuales. Los creadores de la serie House of Cards (Beau Willimon, 2013) privilegian esta estrategia conocida como quiebra de la cuarta pared- desde la primera escena donde se presenta a su, por otro lado, cuestionable protagonista.

Finalmente, cabe plantear el efecto de una última dimensión de la intimidad, la intimidad situacional, inducida por el acceso a momentos privados del personaje. La narración nos permite acceder no solo a sus secretos, sino también a situaciones comprometidas y embarazosas del personaje, fomentando directamente la simpatía hacia este al establecer un vínculo fundado sobre una complicidad e intimidad compartida (Eder, 2006: 72). Se trata de un factor poco estudia-

8 Por PSI se entiende una corriente interesada en la vinculación afectiva del espectador con los personajes mediáticos, si bien desarrollada en el campo de los estudios psicológicos de la recepción televisiva. Las características de los noticiarios y talk shows han orientado sus investigaciones hacia cuestiones diferentes a las que habitualmente ocupan la agenda de las otras teorías, enfatizando la importancia de la apelación directa al espectador por parte de los presentadores, el modo en que el espectador contesta activamente dirigiéndose a la pantalla, el poder de convicción de los media personae sobre los espectadores e incluso las dificultades por las que pasan algunos espectadores para superar la separación cuando el programa deja de emitirse (Klimmt, Hartmann y Schramm, 2011; Eyal y Cohen, 2006; Lathera y Moyer-Gusea, 2011; Branch, Wilson y Agnew, 2013). 
do, que resulta imposible disociar de otros con los que se entreteje -compasión, similitud, familiaridad experiencial-, y que generalmente estimula haciendo que el espectador acceda a un espacio en la vida real vedado tanto por el deseo de privacidad de la víctima como por la consideración del espectador. Las comedias románticas, por ejemplo, recurren habitualmente a esta estrategia estableciendo una sensación de cercanía con sus protagonistas al acompañarlos cuando van al excusado (el propio término alude a la privacidad de lo que allí acontece). Pero probablemente el espacio privilegiado cinematograficamente para movilizar este factor sea la ducha, donde resulta habitual que los personajes laven sus conciencias o lloren sus desgracias.

La paradigmática escena de la ducha de Psycho (Psicosis, Alfred Hitchcock, 1960) juega con este recurso. Tras presentarnos a una protagonista de actitud frívola y licenciosa -al menos para los valores de la época, cotexto- que comete un acto delictivo -un hurto- en las primeras escenas del filme, Hitchcock nos alía progresivamente con ella por diferentes vías. Primero principios débiles, como la atracción y la familiaridad, con su creciente efecto sobre el favoritismo. Pero progresivamente factores más intensos, como la compasión, al enfrentarla a una sucesión de peligros en su huida -el encuentro con su jefe, el interrogatorio del policía, las sospechas del vendedor de coche- que comprometen su éxito, así como la homofilia corporal -tras innumerables primeros planos a través de los cuales, por vía empática, la puesta en escena contagia los estados emocionales del personaje en el espectador-. A mitad de la película el personaje parece decidido a devolver el dinero, un gesto moralmente loable que intensifica su simpatía. Tomada la decisión se introduce en la ducha, siendo el espectador invitado a acompañarla, para así compartir con ella un momento de absoluta privacidad en el cual, creyéndose sola, se presenta absorta en su vulnerabilidad, atrapada entre la culpa, la soledad y el desamparo.

Curiosamente, Hitchcock plantea este pasaje de intimidad compartida como el último escalón de un recorrido narrativo estructurado para que la simpatía se construya lentamente. Pero una vez alcanzado este punto culminante el director liquida al personaje ante los ojos del espectador, produciendo un intenso desgarro emocional que no solo se explica por lo inesperado del evento, o por la muerte de un personaje simpático. Sino porque dicho asesinato desbarata todo el laborioso trabajo de exoneración, comprensión y adhesión progresiva que el espectador se ha visto obligado a hacer para erigir su simpatía, que por fin, en ese instante, parecía definitivamente asentada. Y porque el asesinato se comete precisamente durante un pasaje en el que el espectador se encuentra compartiendo tranquilamente un momento de intimidad cómplice con la protagonista, en el que nadie más pareciera poder entrometerse. De este modo, partiendo de una buscada antipatía, Hitchcock trabaja con todos los recursos a su alcance para estimular lentamente la simpatía hacia su personaje, poniendo en juego prácticamente todas las estrategias aquí presentadas. Y encuentra en la intimidad situacional no solo el factor idóneo con el que rematar su proyecto, sino la condición narrativa perfecta, desde el punto de vista del contrato espectatorial, para despedazarlo. 


\section{Conclusiones}

La vinculación afectiva con los personajes ficcionales es uno de los pilares centrales de la experiencia fílmica, resultando el principal condicionante de la reacción emocional del espectador cinematográfico. Se trata de un fenómeno de genealogía compleja, que depende de la interacción de una multiplicidad de factores interrelacionados entre sí, susceptibles todos ellos de inducir diferentes grados de simpatía/antipatía hacia el personaje. Dada la compleja interacción de los sentimientos que la constituyen, su distinción resulta útil de cara a comprender cómo distintos artefactos narrativos producen diferentes reacciones en espectadores diversos.

El objetivo de este texto ha sido plantear una larga serie de factores implicados en dicho proceso, en un intento de trasdencer los errores o las limitaciones de otros planteamientos al respecto, algunos demasiados ingenuos, dados a valorar en exceso la importancia de factores como las semejanzas sociales (homofilia social) o la alineación perceptivo-cognitiva (familiaridad perceptiva y motivacional) entre espectador y personajes, otros inevitablemente parciales, al enfatizar la importancia de la aprobación moral sin tener en cuenta otros factores que no solo intervienen en el proceso, sino que además modulan dicha aprobación (por vía del favoritismo, así como otros sesgos y cortocircuitos emocionales). Por ello, además de ofrecer un cuadro de factores implicados, se ha procurado respaldar el posible efecto de cada factor haciendo referencia a diferentes investigaciones que las abalan, procedentes de la psicología social, y se han expuesto las diversas relaciones de interdependencia y reciprocidad que tienen lugar entre ellos.

Con ello se espera ofrecer una imagen más detallada de la simpatía hacia los personajes de ficción, incitando a la reflexión no solo sobre su importancia y sus consecuencias narrativas, sino también sobre los complejos procesos implicados en su elicitación, cuyo estudio pormenorizado permite entender mejor la eficacia de cualquier narración en este sentido, pero, sobre todo, examinar adecuadamente los casos donde la vinculación afectiva se ve complicada o alterada progresivamente a lo largo de un relato.

En este sentido, una investigación pormenorizada sobre los recursos con los que una narración estimula y/o cuestiona los diversos factores aquí expuestos permite, en primer lugar, analizar el modo en que las películas organizan sus materiales con el objetivo de asegurar determinadas lecturas preferentes, redundando en los factores positivos con el fin de que la pretendida simpatía hacia el protagonista tenga el mayor alcance posible (y, por tanto, mayores posibilidades de distribución). Ayuda a explicar porqué el espectador simpatiza en diferente grado con los diferentes personajes presentados por una narración, situación especialmente relevante en los casos en que estos personajes se ven enfrentados en la historia. Proporciona un marco adecuado para analizar cómo determinadas obras consiguen que sus espectadores simpaticen con los personajes a pesar de presentar estos características susceptibles de producir su rechazo (la 'simpatía con el diablo', por ejemplo), y cómo la obra modula a lo largo de su metraje dichas simpatías y, en consecuencia, los afectos 
derivados de ellas. Y permite explicar porqué una misma obra es experimentada de forma desigual, en ocasiones completamente contraria, por diferentes espectadores, cuestión de especial relevancia para los estudios (multi)culturales.

\section{Referencias bibliográficas}

Appiah, K. A. (2008): Experiments in Ethics. Cambridge: Harvard University Press.

Aristóteles. (2006): Poética. Madrid:Alianza Editorial.

Aronson, E., Willerman, B., y Floyd, J. (1966): “The effect of a pratfall on increasing interpersonal attractiveness”, Psychonomic Science, 4(6), pp. 227-228.

Auter, P. (1992): “TV that talks back: An experimental validation of a parasocial interaction scale”, Journal of Broadcasting and Electronic Media, 36 (1), pp. 173-181.

Barnett, M. A., Tetreault, P. A., y Masbad, I. (1987): "Empathy with a rape victim: the role of similarity of experience", Violence and Victims, 2 (4), pp. 255-262.

Bateson, D. et al. (2003): “... As you Would have Them Do Unto You”: Does Imagining Yourself in the Other's Place Stimulate Moral Action?”, Personality and Social Psychology Bulletin, 29 (9), pp. 1190-201.

Berreby, D. (2008): Us and Them: The Science of Identity. Chicago: University Of Chicago Press.

Booth, W. (1961): The Rhetoric of Fiction. Chicago: University of Chicago Press.

Boven, L. V. et al. (2013): "Changing Places: A Dual Judgment Model of Empathy Gaps in Emotional Perspective Taking”, Advances in experimental social psychology, 47, pp. 117-171.

Branch, S., Wilson, K. M., y Agnew, C. R. (2013): “Committed to Oprah, Homer, or House: Using the investment model to understand parasocial relationships”, Psychology of Popular Media Culture, 2 (2), pp. 96-109.

Carroll, N. (1996): Theorising the Moving Image. Cambridge: Cambridge University Press.

- (2008): The Philosophy of Motion Pictures. Malden: Blackwell Publishing.

- (2009): “Tony Soprano y nuestra simpatía por el diablo”, en Los Soprano Forever. Antimanual de una serie de culto. Madrid: Errata naturae ediciones, pp. 55-74.

Chartrand, T. L., y Van Baaren, R. (2009): “Human mimicry”, en Olson, J.M, y Zanna, M.P. (eds.): Advances in Experimental Social Psychology. San Diego: Academic Press, pp. 219-274. 
Christy, C. A., y Voigt, H. (1994): “Bystander Responses to Public Episodes of Child Abuse”, Journal of Applied Social Psychology, 24 (9), pp. 824-847.

Cohen, J. (2006): “Audience Identification with Media Characters”, en Bryant, J. y Vorderer, P (eds.): The psychology of entertainment. Mahwah: Erlbaum, pp. 183-197.

Coplan, A. (2009): “Empathy and Character Engagement", en Livingstone, P. y Platinga, C. (eds.): The Routledge Companion to Philosophy and Film. London; New York: Routledge, pp. 97-110.

Eder, J. (2006): “Ways of Being Close to Characters”, Film Studies (8), pp. 68-80.

Efran, M. G. (1974): "The Effect of Physical Appearance on the Judgment of Guilt, Interpersonal Attraction, and Severity of Recommended Punishment in Simulated Jury Task", Journal of Research in Personality, 8, pp. 45-54.

Etcoff, N. (1999): Survival of the Prettiest: The Science of Beauty. New York: Anchor Books.

Eyal, K., y Cohen, J. (2006): “When Good 'Friends' Say Goodbye: A Parasocial Breakup Study”, Journal of Broadcasting \& Electronic Media, 50 (3), pp. 502-523.

Gabriel, U. (2015): “Emotions and legal judgements: normative issues and empirical findings”, en Oswald, M.E., Bieneck, S. y Hupfeld-Heinemann, J. (eds.): Social Psychology of Punishment of Crime. Chichester: Wiley-Blackwell, pp. 157-172.

Gaut, B. (2010): “Empathy and Identification in Cinema”, en French, P.A., Wettstein, H. K. y Saint, M. (eds.): Film and the Emotions. Boston; Oxford: Blackwell, pp. 136-157.

Glocker, M. et al. (2009): "Baby Schema in Infant Faces Induces Cuteness Perception and Motivation for Caretaking in Adults", Ethology, 115 (3), pp. 257-263.

Glover, J. (2001): Humanity: A Moral History of the Twentieth Century. New Haven; London: Yale University Press.

Goldman, A. I. (2006): Simulating Minds: The Philosophy, Psychology, and Neuroscience of Mindreading, Oxford: Oxford University Press. doi:10.1093/0195138929.001.0001.

Gould, S. J. (2010): El pulgar del panda. Madrid: Grupo Planeta.

Haidt, J. (2001): “The Emotional Dog and Its Rational Tail: A Social Intuitionist Approach to Moral Judgment”, Psychological Review, 108 (4), pp. 814-834.

- (2012): The Righteous Mind:Why Good People Are Divided by Politics and Religion. London: Allen Lane.

Haidt, J., y Kesebir, S. (2010). “Morality”, en Fiske, S.T., Gilbert, D.T., y Lindzey, G. (eds.): Handbook of Social Psychology. Hoboken: Wiley, pp. 797-832. 
Huston, T. L. (2013): Foundations of Interpersonal Attraction. New York: Academic Press.

Jones, W. E. (2011): “Partiality and Transgressive Comedy: Making Sense of our Amusement at His Girl Friday", en Jones, W.E. y Vice, S. (eds.): Ethics at the Cinema. Oxford: Oxford University Press, pp. 91-116.

Kahneman, D. (2012): Pensar rápido, pensar despacio. Barcelona: Debate.

Keysers, C. (2011): The Empathic Brain. Social Brain Press.

Klimmt, C., Hartmann, T., y Schramm, H. (2011): “Parasocial Interactions and Relationships”, en Bryant, J. y Vorderer, P. (eds.): Psychology of Entertainment. London: Routledge, pp. 291-313.

Lathera, J., y Moyer-Gusea, E. (2011): "How do we react when our favorite characters are taken away? An examination of a temporary parasocial breakup”, Mass Communication and Society, 14 (2), pp. 196-215.

Loewenstein, G., y Small, D. A. (2007): “The Scarecrow and the Tinman: The Vicissitudes of Human Sympathy and Caring”. Review of General Psychology, 11 (2), pp. 112-116.

Neill, A. (1996): “Empathy (and) Film Fiction”, en Bordwell, D. y Carroll, N. (eds.): Post-Theory: Reconstructing Film Studies. Madison: The University of Wisconsin Press, pp. 175-194.

Nodelman, P. (1991): “The eye and the I: Identification and first-person narratives in picture books”, Children's Literature, 19 (1), pp. 1-30.

Nye, J. (2004). Soft Power: The Means to Success in World Politics. New York: Public Affairs.

Plantinga, C. (1999): “Scene of Empathy and the Human Face on Film”, en Plantinga, C. y Smith, G. M. (eds.): Passionate Views: Film, Cognition, and Emotion. Baltimore: Johns Hopkins University Press, pp. 239-256.

- (2009). Moving Viewers. American Film and the Spectator's Experience. Berkeley: University of California Press.

- (2010). “I Followed the Rules, and They All Loved You More': Moral Judgment and Attitudes toward Fictional Characters in Film”, en French, P. A. y Wettstein, H.K. (eds.): Film and the emotions. Oxford: Wiley-Blackwell, pp. 34-51.

Raney, A. A. (2004): "Expanding disposition theory: Reconsidering character liking, moral evaluations, and enjoyment", Communication Theory, 14 (A), pp. 348-369.

- (2011). “The Psychology of Dispositional-Based Theories of Media Entertainment”, en Bryant, J. y Vorderer, P. (eds.): Psychology of Entertainment. New York; London: Routledge, pp. 137-150.

- (2011). “The Role of Morality in Emotional Reactions to and Enjoyment of Media Entertainment”, Journal of Media Psychology, 23 (1), pp. 18-23. 
Schiller, B., Baumgartner, T., y Knoch, D. (2014): "Intergroup bias in third-party punishment stems from both ingroup favoritism and outgroup discrimination", Evolution and Human Behavior, 35 (3), pp. 169-175.

Small, D. A., y Loewenstein, G. (2003): “Helping a victim or helping the victim: Altruism and identifiabilty”, Journal of Risk and Uncertainty, 26 (1), pp. 5-16.

Smith, M. (1995): Engaging Characters: Fiction, Emotion, and the Cinema. Oxford: Ciadenron Press.

- (1999). “Gangsters, Cannibals, Aesthetes, or Apparently Perverse Allegiances", en Plantinga, C. y Smith, G. M. (eds.): Passionate Views: Film, Cognition, and Emotion. Baltimore: Johns Hopkins University Press, pp. 217-238.

- (2011). “Just What Is It That Makes Tony Soprano Such an Appealing, Attractive Murderer?", en Jones, W.E. y Vice, S. (eds.): Ethics at the Cinema. Oxford: Oxford University Press, pp. 66-90.

Vaage, M. B. (2010): "Fiction Film and the Varieties of Empathic Engagement”, Midwest Studies In Philosophy, 34, pp. 158-179. doi:10.1111/j.1475-4975.2010.00200.x

- (1013): "Fictional Reliefs and Reality Checks", Screen, 54 (2), pp. 218-237.

- (2014): “Blinded by Familiarity: Partiality, Morality and Engagement in Television Series”, en Nannicelli, T. y Taberham, P. (eds.): Cognitive Media Theory. Oxford; New York: Routledge, pp. 268-284.

Wood, J. (2008): How Fiction Works. New York: Straus and Giroux.

Zajonc, R. (2001): "Mere Exposure: A Gateway to the Subliminal”, Current Directions in Psychological Science, 10 (6), pp. 224-228.

Zillmann, D., y Cantor, J. (1976): “A disposition theory of humor and mirth”, en Chapman, A. y Foot H. (eds.): Humor and laughter: Theory, research, and application. London: Wiley, pp. 93-115. 